( $+1 \mathrm{SD}$ ), the predicted probability of having the active disease was $11 \%$ vs. $52 \%$ for those higher vs. lower in SNS ( $\pm 1 \mathrm{SD}$ ), respectively. In comparison, among those lower in ONS (-1SD), the likelihood of active disease was $38 \%$ vs $25 \%$ for those higher vs. lower in SNS, respectively. Thus, among patients with greater math abilities, having greater beliefs in one's abilities appeared beneficial; however, among patients with lower math abilities, greater ability beliefs appeared harmful.

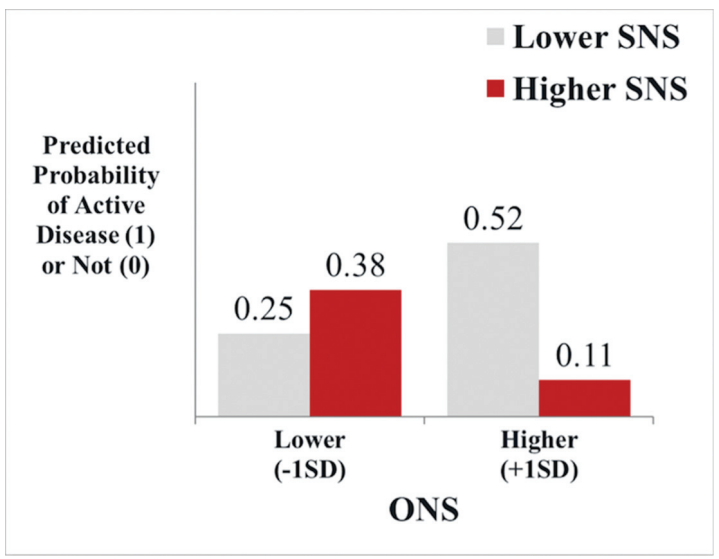

Conclusions: Patients with greater math ability, coupled with greater beliefs in one's numeric ability, were least likely to have the active disease. This is consistent with psychological theory about actual math ability and perceived math ability as an outcome predictor. Patients that are overconfident and more active in their health and numeric tasks may make decisions that lead them to worse outcomes. The SNS and ONS may be an efficient screening tool to identify high risk SLE patients that may require extra health care needs.

Disclosure of Interest: None declared

DOI: 10.1136/annrheumdis-2018-eular.7006

\section{THU0344 ASSESSMENT OF THE PSYCHOMETRIC PROPERTIES OF PATIENT-REPORTED OUTCOMES OF DEPRESSION IN SLE}

${ }^{1}$ A. Kwan, S. Marzouk ${ }^{2}$, H. Ghanean ${ }^{3}$, K. Ali ${ }^{4}$, N. Anderson ${ }^{4}$, J. Su ${ }^{4}$, Z. Touma ${ }^{5}$. ${ }^{1}$ Medicine, Queen's University, Kingston, Ontario; ${ }^{2}$ Department of Psychiatry,

${ }^{3}$ Psychiatry, University of Toronto; ${ }^{4}$ Toronto Western Hospital; ${ }^{5}$ Rheumatology, University of Toronto, Toronto, Canada

Background: Mood disorders, including depression, are amongst the most common manifestations of neuro-psychiatric SLE. Currently, the screening and diagnosis for depression in ambulatory settings are delayed and often missed due to the lack of standardised valid questionnaires for assessing depression in patients with SLE.

Objectives: This study aims to: 1) Determine the prevalence of depression in SLE patients using the Centre for Epidemiological Studies-Depression Scale [CES-D] and Hospital Anxiety and Depression Scale [HADS] questionnaires. 2) Study the criterion validity and interpretability of CES-D and HADS, and 3) evaluate their diagnostic accuracy when compared to the assessment of an independent psychiatric assessment using the Mini-International Neuropsychiatric Interview (MINI), based on the DSM-5, as the gold standard.

Methods: A cross-sectional study of consecutive consenting SLE patients $(n=227)$, aged 18-65 and attending the Toronto Lupus Clinic between June 2017-September 2017, was performed. Participants were screened for depression using the CES-D and HADS, and underwent the MINI on the same date of their clinic visit. Conventional cut-off scores were used to indicate the prevalence of depression: CES-D $\geq 16$ and HADS-D $\geq 8$. Sensitivity, specificity, positive predictive value (PPV), and negative predictive value (NPV) were evaluated against the MINI. Receiver operator characteristic (ROC) curves and the Youden Index were utilised to determine the optimal cut-off scores for CES-D and HADS-D.

Results: Among 227 patients, the prevalence of depression ranged from $27.8 \%$ (HADS-D) to $46.3 \%$ (CES-D). ROC curves showed that the CES-D (AUC 0.86, 95\% Cl: 0.78-0.95) slightly outperformed HADS-D (AUC 0.84, 95\% Cl: 0.750.93), when compared to the MINI. The sensitivity, specificity, PPV, and NPV for CES-D at the optimal cut-off of 26 was $80 \%, 82 \%, 43 \%$, and $96 \%$, respectively. The Youden index exhibited optimal cut-offs for CES-D at 26 and HADS-D at 8 that optimised their sensitivity and specificity as screening metrics for depression in SLE patients. The performance of the CES-D and HADS-D at various cut-offs are displayed in table 1 below.
Abstract THU0344 - Table 1. Cut-offs to predict depression by Youden Index

\begin{tabular}{lcccc}
\hline Cutoffs & Sensitivity & Specificity & PPV & NPV \\
\hline CES-D & & & & \\
$26^{*}$ & 0.80 & 0.82 & 0.43 & 0.96 \\
24 & 0.80 & 0.77 & 0.37 & 0.96 \\
$21^{\dagger}$ & 0.85 & 0.71 & 0.34 & 0.97 \\
$16^{\dagger}$ & 0.95 & 0.55 & 0.27 & 0.98 \\
HADS-D & & & & \\
$8^{* \dagger}$ & 0.80 & 0.79 & 0.40 & 0.96 \\
7 & 0.85 & 0.72 & 0.35 & 0.97 \\
6 & 0.95 & 0.62 & 0.30 & 0.99 \\
\hline
\end{tabular}

*Optimal cutoff as determined by the Youden Index

'Conventional cutoffs found in literature

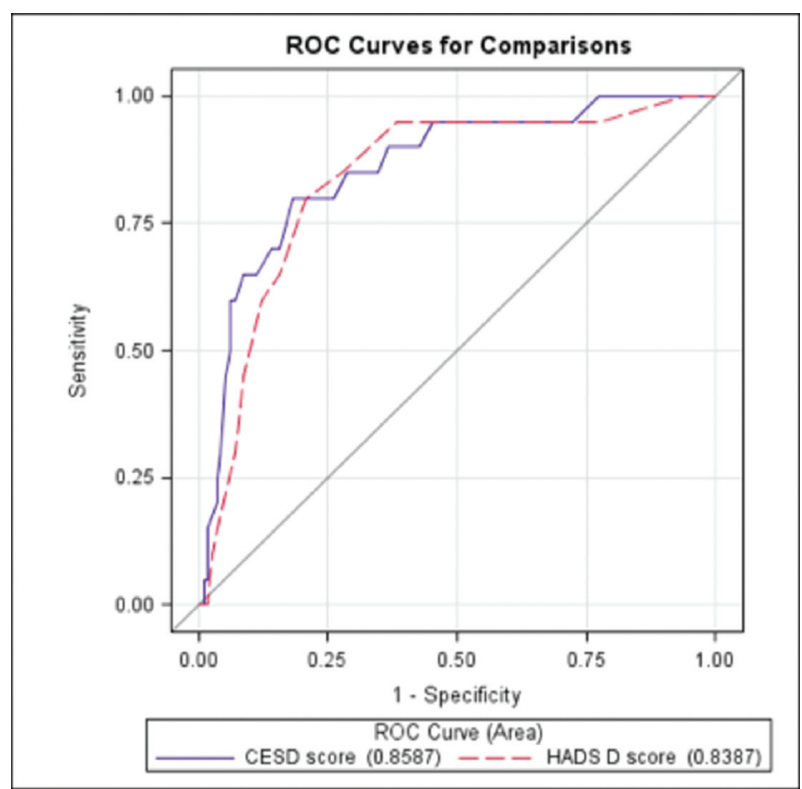

Abstract THU0344 - Figure 1. ROC curves for comparisons

Conclusions: This study assessed the criterion validity and interpretability of patient-reported outcome tools HADS, and CES-D for depression screening in SLE patients. Compared to the gold standard, CES-D outperformed HADS-D. These results suggest that SLE-specific cut-offs may improve diagnostic accuracy of current screening metrics in lupus.

Acknowledgements: The University of Toronto Lupus Clinic is supported by the University Health Network, the Lou Rocca Family, and the Lupus Foundation of Ontario. This summer project is supported by the Canadian Rheumatology Association Summer Studentship Program.

Disclosure of Interest: None declared

DOI: 10.1136/annrheumdis-2018-eular.1094

\section{\begin{tabular}{|l|l}
\hline THU0345 & SENSITIVITY AND SPECIFICITY OF ANTIBODIES
\end{tabular} AGAINST CARBAMYLATED PROTEINS IN A MONOCENTRIC COHORT OF PATIENTS WITH SYSTEMIC LUPUS ERITAMATOSUS AND RHEUMATOID ARTHRITIS}

${ }^{1}$ B. Özdemir, O. Kucuksahin ${ }^{2}$, T.H. Yeşil ${ }^{3}$, S. Akan ${ }^{4}$, S. Erten ${ }^{2} .{ }^{1}$ Internal Medicine, Yılıdırm Beyazıt University, ${ }^{2}$ Rheumatology, Yıldırım Beyazıt University;

${ }^{3}$ Rheumatology; ${ }^{4}$ Internal Medicine, Atatürk Research and Educational Hospital, Ankara, Turkey

Background: Systemic lupus erythematosus (SLE) is a chronic systemic autoim mune disease characterised by numerous organ involvement. In SLE, autoantibody development against nucleic acids and their binding proteins plays an important role in disease pathogenesis.

Objectives: Purpose of this study is the determination of the diagnostic value of anti-carbamyl antibody in patients with Systemic Lupus Erythematosus and rheumatoid arthritis and the relationship with disease prognosis.

Methods: Fifty-seven SLE patients (F/M 50/7; median age 40.9 \pm 13.7 ; median disease duration 2 years) were included in the study according to the 2012 SLICC SLE diagnostic criteria. 46 RA patients (F/M 38/8, median age 54.2 \pm 12.4 years, 